\title{
ESTEROIDES SEXUALES
}

\author{
Dr. Hernando Amaya-León*
}

MENSAJE DEL PRESIDENTE DE LA FUNDACION PARA INVESTIGACIONES

HORMONALES EN LA INAUGURACION DEL SIMPOSIO.

No hay duda de que asistimos a una época de profundas transformaciones en todos los campos de la actividad humana.

El hombre vivió evolucionando durante milenios sujeto a leyes inmutables, relativamente tranquilo y esperando con optimismo el lento pasar de los tiempos.

De pronto, en el siglo XIX de nuestra Era cristiana, hay un despertar activísimo y se inicia una total renovación del saber con marcadas influencias en la filosofía, el arte, la sociología, la economía, la medicina y otras ramas importantes de la tecnología. Por doquier surgen teorías y se inicia la investigación en escala: es el prodromo de algo imprevisto pero inminente.

$Y$ aparece la vigésima centuria, transformista, violenta, explosiva. To. da aquella inercia acumulada durante tanto tiempo por una humanidad tranquila, se pone en ebullición $y$ en un momento dado explota; y se inicia entonces una nueva era de tremenda revolución.

En el campo médico, esto es evidente. Yo creo que lo hecho en los últimos 50 años supera con creces a lo acumulado en muchas centurias anteriores, pero, estoy seguro, es tan solo el prólogo de transformaciones que aún no podemos prever.

Al hablar de Endocrinología estos conceptos encajan con exactitud; su padre, Claude Bernard, precursor de esta novel ciencia, solo surgió a la vida científica a mediados del siglo XIX; años después, Bayliss y Sterling, descubridores de la secretina, introdujeron el término "hormonas"; y fue Knaver en 1900, quien primero demostró la naturaleza endocrina del ovario, pero hasta 1912 logró Adler producir estro en cobayos luego de inyección de extractos ováricos. Por esa época, 1911, Glynn en Estados Unidos establecía la correlación entre la corteza suprarrenal y los problemas del sexo, y Steinach (1913), con gran sentido científico, escribía los primeros ensayos serios sobre los andrógenos, ya enunciados desde 1889 por un profeta intuitivo, Brown Sequard. Así mismo en 1910, el alemán Frankel demostró la propiedad del cuerpo amarillo para la nidación y mantenimiento del embarazo, primer trabajo de investigación en esta hormona desde la sugerencia

Jefe del Departamento de Obstetricia y Ginecología de la Facultad de Medicina, Universidad Nacional. 
del francés Prenant en 1898 de que el cuerpo amarillo del ovario podría ser órgano de secreción interna; pero hasta 1928, Corner, y Allen aislaron la progesterona, y fue Butenandt en 1934 quien hizo las determinaciones precisas de dicha hormona.

Siguiendo este esbozo histórico de los esteroides sexuales, es preciso incluir a Doissy quien en 1923 aisló un potente estrógeno del ovario; a Ascheim y Zondek quienes en 1927 descubrieron la gonadotropina coriónica, a la cual denominaron Prolan; a Browne que en 1930 aisló de la placenta el estriol y a Venning que pudo en 1937 correlacionar la extracción del pregnandiol con la presencia de la progesterona. Las investigaciones de Laqueur, Butenandt, Reichstein, Ruzika y Kendall en los años treinta, fueron los pasos fundamentales del conocimiento de los andrógenos y su relación como esteroides sexuales en el aparato genital femenino.

De entonces acá los avances han sido extraordinarios; al obtener en 1953 la 19-norprogesterona, Tulner y Hertz abrieron el campo de los nuevos progestágenos; para no ser infiel con la historia, tendría que mencionar cientos de nombres, muchos de ellos aún activos y algunos aquí presentes, para decir cuál ha sido el progreso en esta rama de la hormonología y cuánta su influencia en la ciencia médica contemporánea.

Cualquier avance en una rama médica contribuye a mejorar el conocimiento de todo el organismo humano, y por tanto afecta el campo de otras epsecialidades, por distantes que parezcan. Esta interrelación adquiere enorme importancia entre la Endocrinología y la Gineco-obstetricia y no hay duda que todo cuanto atañe a una se refleja necesariamen- te en la otra; $y$, en particular, al hablar de la fisiología de la reproducción, las dos ramas investigativas se confunden en una sola. Por eso el problema de esteroides sexuales reúne a científicos de las dos actividades.

La reproducción constituye carácter fundamental en la conservación de las especies; y es un fenómeno biológico muy complejo.

Como anota el español Botella Llusiá, en la causalidad biológica simple, hay siempre armonía entre la magnitud de la causa y la cuantía de la respuesta. Pero es verdad también que algunas veces la respuesta es desproporcionadamente mayor que la causa. Por estos conceptos, en tratándose de la fisiología de la reproducción, son de extraordinario interés.

El mismo autor anota que en "todo proceso morfogenético, tanto sea éste el desarrollo embrionario como el crecimeinto en el individuo joven - como la reproducción en el adulto, tenemos que distinguir dos elementos fundamentales: una causalidad determinada directamente por las combinaciones genéticas de la herencia y una condicionalidad determinada humoralmente por el ambiente químico o físico-químico en que la causalidad primitiva se desenvuel$v e^{\prime \prime}$.

En el intenso problema que hoy vivimos en relación a la angustia que determina el aumento desproporcionado de la población humana, la ciencia médica intenta contribuir a detener ese fenómeno explosivo actuando sobre esa condicionalidad biológica; y lo quiere hacer a través de sus catalizadores específicos o biocatalizadores. De los cuatro grupos descritos hoy -hormonas, enzimas, vitaminas y organicinas - se le 
ha concedido privilegio al primero, quizá por ser el más conocido, o mejor, por ser el menos desconocido. Además, las hormonas constituyen el biocatalizador más específico y más definido en su misión en el organismo humano.

Tres son las hormonas básicas de este grupo, o esteroides sexuales: estrógenos, progestágenos y andrógenos; y todos ellos siempre presentes tanto en el hombre como en la mujer. Es aquello que algunos han querido denominar hermafroditismo bioquímico; punto evidente y simple que no podremos olvidar en el curso de investigaciones sobre el tema; así como es trascendental no perder de vista que la verdadera acción característica de esos esteroides depende en gran parte de la respuesta del órgano efector.

Estos elementales conceptos aplicados fielmente en el desarrollo ortofilogenético del ser humano, debe hacernos meditar en lo que respecta a cada caso en particular; porque no podemos saber que todos los órganos efectores respondan siempre de igual manera cuando se administran esteroides sexuales en dosis que superen los niveles considerados como normales.

En la anovulación yatrogénica tengo la impresión de que se ha querido valorar a la humanidad con un patrón estandar, y me temo, por eso, que los efectores no estén respondiendo en forma similar. Si bien es cierto que estamos obteniendo el primer objetivo de nuestros propósitos, regulación de la fertilidad, no estamos muy convencidos de sus consecuencias futuras en muchos aspectos. La individualidad kijológica está básicamente cimentada en el equilibrio psiconeuroendocrino; ignoramos cuándo y cómo ese balance se puede romper, y lanzar entonces a un organismo a la anarquía celular o a la alteración funcional más - menos irreversible o, lo que sería peor, a la aparición de seres mentalmente muy distintos al patrón actual.

Hablando de anarquía celular, desde Lacasagne (1923), se viene pensando en la relación directa entre hormonas esteroides y cáncer; cuando aquel investigador francés produjo tumor maligno mamario en ratones tratados con esteroides sexuales, se creó una mentalidad muy precisa a ese respecto, confirmada luego por muchos autores: en 1940 Allen, Gardner y luego Nathanson en 1947, Lipschutz en 1950, entre otros, con la advertencia, claro está, de que la evaluación de los factores endocrinos en la etiología del cáncer humano era muy difícil de establecer con precisión, por cuanto los regímenes experimentales no pueden ser hechos en condiciones iguales a los de animales; pero basados en datos estadísticos, clínicos y terapéuticos, se sostuvo con mucho fervor que los esteroides sexuales eran muy sugestivos de influir en la etiología del cáncer.

Al surgir esta nueva era de los anovulatorios, en menos de 20 años, los aspectos conceptuales a ese respecto están cambiando. Las estadísticas demuestran ausencia de efectos carcinogenéticos y los resultados terapéuticos sugieren acción benéfica en algunos tipos de tumor maligno; es decir, todo lo contrario a lo imaginado en la veintena inmediatamente anterior.

Sería aventurado afirmar cuál tendencia tiene la razón; pero creo que se está llevando a cabo la más grande experiencia clínica que jamás haya emprendido la raza humana; y no 
podrá decirse nada definitivo antes de que transcurran siquiera tres generaciones.

Buenos temas para próximos simposios sobre esteroides sexuales podrían ser su influencia en genética, investigaciones en cáncer, y precisión de interrelaciones con el resto del árbol endocrino.

La investigación, sin duda alguna, constituye una de las misiones primordiales de toda institución universitaria. Mientras la actividad investigadora permanezca ausente o se halle en fase embrionaria, ha de convenirse que el objetivo de los centros de cultura superior no ha alcanzado su completa plenitud.

En Colombia, al igual que en la mayoría de las naciones en vía de desarrollo, la investigación ha venido haciéndose de manera tímida y balbuciente. Nuestra universidad estatal, reflejo de nuestro país, aún no ha superado la etapa esencialmente práctica, utilitarista, pues sus precarios recursos económicos son apenas suficientes para entregar conocimientos pero no para sufragar empresas dedicadas a las disciplinas creadoras.

Sin embargo, los estamentos directivos y docentes han adquirido conciencia de la urgencia inaplazable de emprender labores investigadoras. El Departamento de Obstetricia y Ginecología de la Universidad Nacional, por ejemplo, creó en 1965 un comité encargado de estudiar, coordinar y financiar sus incipientes programas de investigación. Por afortunada coincidencia algunos meses más tarde llegó a Bogotá un grupo destacado de funcionarios de la casa farmacéutica Schering A. G. Berlín, quienes viajaban por Latinoamérica observando los distintos ambientes médicos universitarios, con miras a otorgar ayuda financiera a aquellos que estuvieran en disposición de poder desarrollar inicialmente investigaciones de orden clínico, pero con posibilidades y proyecciones futuras mucho más amplias. Gracias a la mediación del doctor Guillermo López Escobar, nos fue ofrecido apoyo. Contando con éste y con el concurso del Centro de Investigaciones de la Universidad Nacional, a la sazón recién creado, nació la Fundación para Investigaciones Hormonales.

Al constituirla legalmente $-\mathrm{y}$ de ello se cumplen ya dos años- quedó establecido en sus estatutos que la Fundación es una entidad de beneficio común, dedicada a la actividad científica y sin ánimo alguno de lucro. Se quiso que su organización fuera académica y autónoma, a fin de evitar interferencias que pudieran desvirtuar la primigenia intención, y, además, se le dejó en libertad de poder recibir eventuales auxilios de cualesquiera personas 0 entidades interesadas en los diferentes campos de la investigación médica. Su Comité Directivo quedó conformado por siete miembros, así: cinco profesores de la Facultad de Medicina, un delegado del Ministerio de Salud Pública y el médico jefe del departamento científico de la Química Schering Colombiana, en representación de la casa patrocinadora.

Luego de esta necesaria presentación de lo que es la Fundación para Investigaciones Hormonales, queremos dejar testimonio expreso de nuestra gratitud hacia Schering A. G. Berlín, sin cuyo generoso aporte no hubiera sido posible llevar a la realidad el anhelo de incrementar la investigación dentro de un sector de nuestra universidad. Precisamente, la realización de este Simposio es de- 
mostración de que ya comienzan a cosecharse los primeros frutos.

Mankeliunas define Simposio como "una reunión de especialistas en una rama de la ciencia, con supuesta igualdad de conocimientos científicos e intereses, que discuten sobre un tema determinado de su especialización", agregando luego que "no hay público asistente porque se trata de un acto esencialmente académico". En esta ocasión la Fundación para Investigaciones Hormonales ha buscado cumplir esos postulados.

Quizá no pueda hablarse de estricta igualdad de conocimientos científicos entre todos nuestros invitados, pero sabemos de su constante interés en lo relacionado a esteroides sexuales. Por ello hemos pedido el aporte de notables conferencistas: Europa, Norte y Latinoamérica están magníficamente representados. Los trabajos cortos demuestran el esfuerzo de quienes valoran el interés de estos conocimientos. A todos ellos anticipo nuestros agradecimientos por su colaboración.

El gobierno colombiano conciente de la importancia de esta reunión, se hace presente en las sesiones de inauguración y clausura con sus más altos funcionarios representativos de la salud y la educación.

A los señores Ministros Ordóñez y Betancourt, la Fundación agradece su gentileza y colaboración.

Los científicos colombianos invitados a este Simposio representan el más alto nivel de nuestras universidades y entidades asistenciales; la Fundación para Investigaciones Hormonales anhela para el futuro la oportunidad de integrar un esfuerzo a través de todo el país con el fin de realizar investigaciones importantes en el campo de la endocrinología gineco-obstétrica; quizá en un día no lejano, este deseo podría ampliarse a países de nuestro fraternal conjunto latinoamericano.

Finalmente, el Comité Directivo de la Fundación para Investigaciones Hormonales, presenta a todos sus invitados un cordial saludo de bienvenida y la seguridad de que pueden considerar esta ciudad como su propia casa. 